\title{
Habilidades Sociais na Agorafobia e Fobia Social ${ }^{1}$
}

\author{
Michelle Levitan ${ }^{2}$ \\ Bernard Rangé \\ Antonio E. Nardi \\ Universidade Federal do Rio de Janeiro
}

\begin{abstract}
RESUMO - Os transtornos ansiosos são freqüentemente associados ao déficit de habilidades sociais (HS). A fobia social é o quadro mais relacionado a esse déficit, enquanto a agorafobia é desconsiderada. O objetivo deste artigo é investigar essa associação por meio de uma revisão da literatura. Foi realizada uma busca eletrônica nas bases de dados PsycINFO, MEDLINE e SCIELO, além das referências dos trabalhos selecionados. O déficit de HS mostrou-se mais presente nos estudos que avaliaram o desempenho de indivíduos socialmente ansiosos em tarefas não estruturadas. A agorafobia parece estar associada a um déficit de assertividade, embora poucos estudos tenham sido conduzidos.
\end{abstract}

Palavras-chave: ansiedade; performance; assertividade.

\section{Social Skills in Agoraphobia and Social Phobia}

\begin{abstract}
Anxiety disorders are commonly associated to social skills deficits. Social phobia is often related to this deficit, while agoraphobia is not considered. The purpose of this review is to investigate the literature on this association. An electronic search was conducted in the following databases: PsycINFO, MEDLINE and SCIELO, besides the references of some selected articles. Social skills deficit were more frequent in the studies that evaluated the performance of anxious individuals on unstructured tasks. Agoraphobia appears to be associated to an assertiveness deficit, although few studies were conducted.
\end{abstract}

Key words: anxiety, performance; assertiveness.

As habilidades sociais (HS) caracterizam-se pela existência de diferentes classes de comportamentos sociais presentes no repertório do indivíduo para lidar de maneira adequada com as demandas das situações interpessoais (Del Prette \& Del Prette, 2001). Assim, é a habilidade de se comunicar e interagir com outros de uma maneira eficaz e apropriada, considerando-se o contexto sócio-cultural vivido, uma vez que ser socialmente hábil em um lugar, pode não ter o mesmo significado em outro (Caballo, 2003).

São divididas em duas categorias, visando principalmente à avaliação dos comportamentos. A categoria molar é a habilidade geral, como fazer e aceitar elogios, fazer pedidos, expressar agrado e desagrado, iniciar conversações, defender os próprios direitos, recusar pedidos, desculpar-se, entre outros. Por sua vez, a categoria molecular é caracterizada por elementos constituintes da molar, como o contato visual, gestos, volume de voz e postura, os quais são medidos de forma mais objetiva (Caballo, 2003).

Em muitos estudos, um dos componentes das HS mais explorado é a assertividade. Lange e Jakubowski (1976) a definem como um estilo de comunicação que contém como base a reivindicação dos próprios direitos e a expressão de pensamentos, sentimentos e crenças de maneira direta, honesta e apropriada que não viole o direito de outras pessoas.

1 Apoio do Conselho Nacional de Desenvolvimento Científico e Tecnológico. Processo número: 554411/2005-9.

2 Endereço: Rua Dona Delfina 28/702, Tijuca, Rio de Janeiro, RJ, Brasil 20511-270. E-mail: milevitan@hotmail.com
Vários estudos associam o déficit em HS a transtornos psiquiátricos. A fobia social (FS) é comumente mais associada devido à natureza do quadro estar baseada nas dificuldades relacionadas às interações sociais, levando alguns autores a aventarem a hipótese de os fóbicos sociais não possuírem um repertório adequado de HS. Outros transtornos psiquiátricos como a agorafobia (Chambless, Hunter \& Jackson, 1982), esquizofrenia (Patterson, Moscona, McKibbin, Davidson \& Jeste, 2001; Chien \& cols, 2003) e a depressão (Segrin, 2000) também estão presentes em estudos de avaliação de HS.

Indivíduos com déficits em HS apresentam uma qualidade de vida bastante comprometida. Os déficits nessas habilidades parecem estar correlacionados com um fraco desempenho acadêmico, delinquiência, abuso de drogas, crises conjugais e desordens emocionais variadas, como transtornos de ansiedade (Del Prette \& Del Prette, 2002). O objetivo do presente trabalho é realizar uma revisão da literatura sobre a possível relação do déficit em HS com a agorafobia e FS. Decidimos avaliar esses dois diagnósticos de transtorno de ansiedade por nos parecerem díspares em relação à abordagem clínica e teórica de HS: a fobia social é considerada e apresentada como um transtorno de ansiedade com claro déficit de HS. Por outro lado, a agorafobia, complicação freqüente do transtorno de pânico, é desconsiderada na abordagem de déficit de HS.

\section{Método}

Foi realizada uma busca eletrônica da literatura nas bases de dados PsycINFO, MEDLINE e SCIELO. O limite de tempo não foi considerado e foram utilizados os seguintes 
termos de procura: social skills, performance, social anxiety, agoraphobia e assertiveness. Foram incluídos artigos que avaliassem as HS em pacientes com ansiedade social, independente da intensidade, e agorafobia. As referências dos trabalhos selecionados também foram utilizadas como fonte de consulta.

\section{Agorafobia e habilidades sociais}

O termo "agorafobia" significa medo de lugares abertos. Na prática clínica, designa medo de sair de casa ou de situações em que o socorro imediato não é possível. O termo, portanto, refere-se a um grupamento inter-relacionado e frequientemente sobreposto de fobias que abrangem o medo de sair de casa, entrar em lugares fechados (aviões, elevadores, cinemas etc.), multidões, lugares públicos, permanecer em uma fila, viajar de ônibus, trem ou automóvel, distanciar-se de casa e estar só em uma dessas situações. A agorafobia é uma complicação freqüente no transtorno de pânico, em que todas as situações temidas têm em comum o medo de passar mal e não se obter socorro fácil ou imediato (Marks \& Gelder, 1966).

Segundo Golstein e Chambless (1978), os pacientes com agorafobia se apresentam como não-assertivos e com crenças sobre uma baixa eficácia pessoal, que é exacerbada quando o ataque de pânico ocorre, levando a pessoa a ficar dependente de outros e evitar as situações ansiogênicas. Assim, o déficit em assertividade vem sendo apontado como uma possível característica presente em grande parte dos agorafóbicos. Uma justificativa para esta investigação estaria no fato de que nem todos os pacientes agorafóbicos se privilegiariam igualmente das técnicas de exposição ao vivo, considerada um método de bastante eficácia no seu tratamento (Emmelkamp \& Kuipers, 1979). Assim, poderiam existir outras características prevalentes nesses pacientes, como a falta de assertividade, que não estariam sendo abordadas, diminuindo a probabilidade da eficácia no tratamento. Os estudos encontrados não avaliaram a prevalência de assertividade em pacientes com agorafobia, porém a compararam entre um grupo clínico e controle, assim como seu impacto no tratamento.

Ball, Otto, Polack e Rosenbaum (1994) avaliaram, em um estudo longitudinal, os preditores da ocorrência de depressão após o tratamento farmacológico e comportamental de 90 pacientes com transtorno do pânico durante dois anos. Pacientes com baixa assertividade apresentaram 4,5 vezes mais risco de desenvolver depressão do que os pacientes assertivos, caracterizando-se como o preditor mais poderoso na associação do transtorno do pânico e depressão, apontando para a importância do treino em habilidades sociais estar inserido no tratamento psicológico do transtorno do pânico.

Chambless (1985), ao avaliar aspectos psicopatológicos de 378 pacientes agorafóbicos com ataques de pânico por questionários de auto-aplicação, percebeu que a evitação comportamental era mais significativa em pacientes que relatavam ter medo de avaliações negativas, menos assertivos e introvertidos. Também encontrou nesses pacientes, uma correlação entre ataques de pânico e depressão, na qual quanto maior a depressão maior a tendência de ser socialmente ansioso, possuir medo de críticas e ser menos assertivo.

Em um estudo realizado somente com agorafóbicos não-assertivos submetidos a três formas de tratamento psicológico, a exposição se mostrou mais eficaz para a ansie- dade e evitação, o treino assertivo teve o melhor resultado no aumento do repertório assertivo e a combinação dos dois produziu resultados comparáveis ao tratamento com exposição. Os autores consideram que, devido à existência de uma discreta melhora dos sintomas fóbicos com o treino assertivo, a agorafobia pode ser parcialmente resultante da não-assertividade (Emmelkamp, Hout \& Vries, 1983).

Chambless e cols. (1982) compararam a assertividade e a ansiedade social em 112 estudantes universitários e 79 pacientes com agorafobia, por meio de questionários específicos. Os participantes agorafóbicos se mostraram significativamente mais ansiosos socialmente e menos assertivos, sugerindo-se que essas características parecem fazer parte da personalidade de indivíduos com esse quadro.

Emmelkamp (1980) avaliou a influência dos problemas interpessoais no tratamento de exposição ao vivo de 17 pacientes agorafóbicos, divididos entre problemas conjugais e assertividade. Quase não houve diferenças entre os resultados do tratamento dos dois grupos. Participantes com alta e baixa assertividade tiveram a mesma melhora, assim como os de alta e baixa satisfação conjugal. Este estudo sugere que, mesmo havendo um nível de assertividade baixo entre os agorafóbicos, não há interferência negativa no tratamento.

Fisher e Wilson (1985), ao investigarem características psicológicas de agorafóbicos que procuravam tratamento, encontraram traços de personalidade como déficit na assertividade de participantes agorafóbicos comparados aos participantes não-agorafóbicos, a partir da aplicação de uma escala de assertividade.

\section{Fobia social e habilidades sociais}

Indivíduos com FS apresentam um medo acentuado e persistente de serem vistos comportando-se de maneira humilhante por meio da demonstração de ansiedade ou de desempenho inadequado. A exposição às situações temidas quase sempre provocam sintomas como sudorese, palpitações, tremores, rubor, entre outros. Algumas situações evitadas são falar em público, urinar em banheiro público, comer e beber na frente de outros e interagir com pessoas (Marks \& Gelder, 1966).

Já é bastante descrito na literatura que fóbicos sociais possuem menos interações sociais do que a maioria dos indivíduos. Logo, possuem menos amigos, namoros e relações sexuais, comparados à população geral ou a pacientes com outros transtornos ansiosos (Alden \& Taylor, 2004; Turner, Beidel, Dancu \& Keys, 1986).

Durante bastante tempo, o déficit em HS foi apontado como o vilão dos pacientes com FS, porém atualmente alguns autores consideram que o fracasso em utilizar as HS adequadas não significa uma incapacidade de exibir o desempenho esperado para aquela situação, mas reflete uma inibição consecutiva à ansiedade excessiva (Rapee \& Heimberg, 1997). Rapee e Spence (2004) aventam a hipótese de o déficit em HS estar mais associado ao desenvolvimento e manutenção da FS em crianças, já que os adultos fatalmente aprenderiam as HS compensatórias para sobreviver às situações sociais. Devido à falta de resultados conclusivos sobre essa associação, alguns autores consideram a possibilidade das HS serem a causa ou a consequiência da FS (Thompson \& Rapee, 2002). 
Os primeiros modelos explicativos da FS baseavam-se na hipótese de que o indivíduo com esse quadro possuiria um repertório comportamental inadequado resultante de um não-aprendizado, levando-o a não se adequar às demandas do ambiente, experenciando uma ansiedade extrema e respostas negativas (Curran, 1977; Segrin \& Flora, 2000). Assim, focavam-se basicamente nos déficits em HS como preditores do desenvolvimento da FS.

Os modelos atuais são mais complexos e enfatizam principalmente os aspectos cognitivos envolvidos no transtorno (Beck, Emery \& Greenberger, 1985; Lucock \& Salkovskis, 1988). Nesse referencial, indivíduos socialmente ansiosos antecipariam negativamente a experiência de interação social, assim como os resultados advindos dessa interação. Crenças sobre serem rejeitados pelos outros a partir de seu desempenho ou passarem por constrangimentos, os deixariam hipervigilantes a sinais que pudessem estar indicando uma ansiedade visível aos outros, contribuindo para a formação do ciclo que mantém a FS. Também consideram importantes na manutenção do quadro, os comportamentos evitativos e de segurança que objetivam diminuir ou eliminar o desconforto provocado pela exposição (Kashdan \& Herbert, 2001).

Ainda é difícil definir se os fóbicos sociais possuem realmente déficit em HS, principalmente devido à mistura de resultados positivos (Monti, Curran, Corriveau, DeLancey \& Hagerman, 1980; Turner, Beidel, Cooley, Woody \& Beidel, 1994) e negativos (Heimberg \& cols., 1990) advindos do treinamento em assertividade para esses pacientes. Assim, pesquisas vêm sido conduzidas, aventando a hipótese do desempenho do indivíduo ser afetado pelo déficit em HS, comparando-se o desempenho em tarefas específicas que envolvam a utilização do repertório de HS entre grupos de pacientes socialmente ansiosos (SA), pouco ansiosos socialmente (PAS), muito ansiosos socialmente (MAS) e grupos controle.

Todos os estudos encontrados foram constituídos de uma etapa naturalística, na qual os participantes são observados e filmados em sua rotina, e, ao realizarem tarefas relativas à interação e desempenho, são avaliados por observadores independentes, segundo as HS apresentadas, além do preenchimento de escalas específicas ao objetivo do estudo.

Thompson e Rapee (2002) realizaram um estudo com 26 estudantes SA e 24 estudantes PAS com o objetivo de avaliar os seus desempenhos em uma tarefa estruturada e não-estruturada. Avaliadores independentes e cegos para o objetivo da tarefa graduaram os comportamentos molares e moleculares em uma escala desde um alto nível de HS até uma extrema inabilidade social nas duas tarefas.

As duas tarefas foram realizadas com a presença de um colaborador do estudo e de um participante por vez. Na primeira parte (tarefa não-estruturada), o participante e o colaborador foram instruídos a aguardarem na mesma sala pelo experimentador, sendo filmados e graduados de acordo com os comportamentos apresentados na interação ocorrida espontaneamente. A segunda tarefa consistia de uma instrução dada pelo experimentador, após a sala de espera, em que os participantes deveriam imaginar-se em uma festa e tentarem se conhecer ao máximo possível em cinco minutos.

Os autores obtiveram como resultado que mulheres MAS apresentaram pior desempenho na tarefa não-estruturada, comparadas às mulheres PAS, porém não houve diferenças entre o desempenho global dos dois grupos na tarefa estruturada. Parece que a estrutura presente nas interações sociais proporciona uma diferenciação dos desempenhos entre pessoas sem FS e com ansiedade social. Assim, em situações em que não há regras claras sobre o desempenho a ser exibido e a demanda ambiental, indivíduos SA tendem a apresentar um desempenho pior do que não-ansiosos.

Pilkonis (1977), um dos primeiros autores a realizar esse estudo comparativo, obteve os mesmos resultados ao avaliar o desempenho de 24 indivíduos com PAS e 22 SA em duas tarefas. $\mathrm{O}$ autor encontrou um desempenho semelhante em ambos os grupos de participantes na tarefa estruturada de discursar para uma platéia, entretanto encontrou diferenças significativas na situação de interação espontânea em uma sala de espera, na qual o grupo MAS teve um desempenho mais pobre do que o SA.

Em um estudo com 26 participantes de ambos os sexos, SA e grupo controle, os autores visaram a observar as HS presentes em algumas situações e encontraram diferenças na interação com o sexo oposto. O grupo SA foi percebido de uma maneira global, como não tão socialmente hábil, e os homens do grupo SA apresentaram menos contato visual comparado ao grupo controle e às mulheres do grupo SA. No discurso a ser apresentado, os avaliadores independentes julgaram ser menos hábeis socialmente os participantes SA (Beidel, Turner \& Dancu, 1985).

Wenzel, Graff-Dolezal, Macho e Brendle (2005) avaliaram diferenças nas HS no contexto das relações amorosas entre um grupo fóbico social e um controle e seus respectivos parceiros, sendo instruídos a terem três tipos de conversação relacionadas ao casal (neutra, negativa e positiva). Os participantes ansiosos demonstraram HS molares pobres ao exibirem mais comportamentos sociais extremamente negativos (p.ex. culpar o outro) na conversa sobre problemas e poucas HS positivas (p.ex. elogiar) durante toda a conversação e não apresentaram 10 dos 11 das HS moleculares desejadas para uma interação.

Spence, Donavan e Toussaint (1999) realizaram um estudo amplo com crianças por meio de questionários sobre HS a serem preenchidos pelos pais, colegas e os próprios alunos e da investigação da interação de 27 crianças entre 7 e 14 anos com diagnóstico de FS e um grupo controle por meio de observação de suas rotinas escolares dentro de sala de aula e no playground por avaliadores. Os autores obtiveram como resultado que crianças com FS participavam de menos interações e apresentavam menor probabilidade de obterem resultados positivos da interação com os colegas, sendo classificados como menos hábeis socialmente pelos seus pais e colegas e apresentando menos repostas assertivas nos questionários.

Por sua vez, Cartwright-Hatton, Tschernitz e Gomersall (2005) encontraram resultados diferentes em um estudo com 40 crianças PAS e MAS com idades entre 10 e 12 anos. As crianças foram informadas de que um adulto iria se juntar a elas, e que deveriam manter pelo menos três minutos de conversação com ele. Avaliadores graduaram as interações e não foram capazes de distinguir os dois grupos, aparentando indicar que não haveria déficits em HS visíveis aos avaliadores. Em outro estudo realizado por Cartwright-Hatton, Hodges e Porter (2003), as crianças foram instruídas a proferir um discurso de dois minutos para uma câmera. Além de aparentarem mais nervosismo, não foi observada nenhuma diferença no desempenho no grupo SA e o controle. 
Alfano, Beidel e Turner (2006) avaliaram o desempenho de 50 crianças e adolescentes com FS e 30 crianças e adolescentes controles em cinco tarefas de role-playing (p.ex. manter uma conversa) realizadas em pares de acordo com a idade dos participantes. Na tarefa relacionada a ler uma história em voz alta, o grupo adolescente com FS foi julgado como menos hábil socialmente do que o grupo controle infantil e as crianças com FS demoraram mais a responder às perguntas referentes a uma tarefa do role-playing do que os grupos controle e o grupo adolescente com FS.

Em outro estudo, 28 pacientes fóbicos sociais e 33 participantes não-clínicos foram instruídos a discursar sobre um tópico de sua preferência durante três minutos. Não foram encontradas diferenças nas avaliações dos observadores sobre o desempenho dos dois grupos (Rapee \& Lim, 1992), sugerindo que participantes com FS não diferem de participantes não-clínicos em relação a déficit em HS.

Strahan e Conger (1998) investigaram o desempenho de 26 participantes do sexo masculino PAS e 27 MAS, por meio de um procedimento singular entre esses estudos. Eles foram instruídos a responder às perguntas da entrevistadora, que não conhecia o objetivo do estudo e havia sido treinada a ser uma entrevistadora moderada, comportando-se de maneira amigável. Ela realizou 10 perguntas destinadas a encorajar a expressão livre de idéias (p.ex. quais são seus pontos fracos?/ como lida com conflitos no trabalho?). Não houve diferenças entre os desempenhos segundo avaliadores independentes. Os autores consideram que as perguntas possam não ter sido difíceis o suficiente para ocasionar diferenças, assim como essa tarefa mostrou-se bem mais estruturada do que pedir para alguém iniciar conversas ou pensar em tópicos interessantes. Desse modo, há uma discrepância no procedimento utilizado em relação aos outros estudos, tornando-se difícil a comparação com os outros resultados encontrados.

\section{Discussão}

Os poucos estudos sobre HS e agorafobia realizados, com exceção de Emmelkamp (1980), parecem apontar para uma associação entre o déficit em assertividade e agorafobia, embora a relação entre a assertividade e a agorafobia ainda não esteja muito clara. Hipóteses aventam que o indivíduo com agorafobia dependeria dos demais para realizar favores, assim se apresentando como mais frágeis, inseguros e dependentes ou que alguns indivíduos teriam traços de personalidade que favoreceriam um prejuízo de assertividade.

Ainda não existem estudos que avaliem um maior repertório de HS que não inclua só a assertividade, assim como estudos de prevalência que possam avaliar essa característica em uma ampla amostra de participantes. Apesar de alguns autores aventarem a possibilidade de o déficit em assertividade fazer parte da "personalidade agorafóbica", o baixo número de trabalhos realizados é um obstáculo a hipóteses mais definitivas.

Apesar da ampla abrangência de trabalhos realizados sobre fatores envolvidos no desenvolvimento da FS, há poucos resultados conclusivos sobre a complexidade desse transtorno. O déficit em HS é encontrado em alguns estudos, influenciando o desempenho do indivíduo socialmente ansioso e, em outros, como um fator inexistente. Alguns artigos dão maior ênfase aos aspectos cognitivos ou de discrepância entre o desempenho real do participante e sua auto-avaliação.

Todos os estudos avaliados utilizaram a metodologia de role-playing estruturado e/ou interação espontânea com um participante treinado. Segundo Bellack (1983), esses tipos de procedimento muitas vezes são problemáticos devido à grande variabilidade de comportamento a ser mensurado, o procedimento a ser adotado e o nível de observação (molar ou molecular). Logo, para uma melhor análise, alguns estudos deveriam conter esses três componentes em comum, o que não foi observado na nossa busca bibliográfica. Esse problema se reflete no artigo de Strahan e Conger (1998), no qual utilizaram um procedimento não encontrado em nenhum outro estudo, inclusive admitindo falhas na elaboração das perguntas envolvidas no role-playing. Assim, torna-se difícil compararmos esses resultados, já que esse instrumento levanta dúvidas.

O conjunto de estudos analisados encontrou uma correlação entre déficit em HS e FS nas situações não-estruturadas de desempenho e interação (Spence \& cols., 1999; Thompson \& Rapee, 2002). Na maioria dos estudos baseados nas tarefas estruturadas, os indivíduos SA não exibiram diferenças nos desempenhos comparados aos participantes sem o transtorno. Rapee e Heimberg (1997) acreditam que os fóbicos sociais tendem a exibir mais déficits em HS em situações não-estruturadas, sugerindo que a estrutura da tarefa parece ser uma variável determinante na exibição das HS. Situações que envolvam mais regras definidas socialmente (p.ex., discurso) parecem proporcionar menos diferenças no desempenho social entre fóbicos sociais e outros grupos do que situações que envolvam regras não definidas (p.ex., festa).

O estudo de Spence e cols (1999) evidencia que o déficit em HS já ocorre na idade infantil, levando ao fracasso nas situações sociais, o que resulta em pensamentos negativos e baixa expectativa sobre o seu desempenho e a reação dos outros, levando as crianças a se isolarem e evitarem essas interações. Essas dificuldades em possuir relacionamentos com colegas, descritas desde o primário, estão associadas a um mau ajustamento na adolescência e na idade adulta.

Esses resultados reforçam a hipótese de que indivíduos ansiosos socialmente sofrem de déficit em HS, porém a relação entre esse déficit e a ansiedade social ainda não é bem entendida. Sem estudos longitudinais, não é possível identificar os fatores antecedentes da FS. Resultados mais conclusivos sobre a associação da agorafobia e FS mostram-se de suma importância na elaboração de protocolos de tratamento. Se for detectado um déficit em HS em pacientes com FS, o treino em HS irá produzir os melhores resultados, logo deve ser considerado como o tratamento padrão para esse quadro.

\section{Referências}

Alden, L. E. \& Taylor, C. T. (2004). Interpersonal processes in social phobia. Clinical Psychology Review, 24(7), 857-882.

Alfano, C. A., Beidel, D. S. \& Turner, S. M. (2006). Cognitive Correlates of Social Phobia Among Children and Adolescents. Journal of Abnormal Child Psychology, 34(2), 189-201.

Ball, S. G., Otto, M. W., Polack, M. H. \& Rosenbaum, J. F. (1994). Predicting prospective episodes of depression in patients with panic disorder: a longitudinal study. Journal of Consulting and Clinical Psychology, 62(2), 359-365. 
Beck, A. T., Emery, G. \& Greenberger, R. L. (1985). Anxiety and phobias: a cognitive perspective. New York: Basic Books.

Beidel, D. C., Turner, S. M. \& Dancu, C. V. (1985). Physiological, cognitive and behavioral aspects of social anxiety. Behaviour Research and Therapy, 23(2), 109-117.

Bellack, A. S. (1983). Recurrent problems in the behavioral assessment of social skill. Behaviour Research and Therapy, 21(1), 29-41.

Caballo, V. E. (2003). Manual de avaliação e treinamento em habilidades sociais. São Paulo: Santos.

Cartwright-Hatton, S., Tschernitz, N. \& Gomersall, H. (2005). Social anxiety in children: social skills deficit, or cognitive distortion? Behaviour Research and Therapy, 43(1), 131-141.

Cartwright-Hatton, S., Hodges, L. \& Porter, J (2003). Social anxiety in childhood: the relationship with self and observer rated social skills. Journal of Child Psychology and Psychiatry, 44(5), 737-742

Chambless, D. L., Hunter, K. \& Jackson, A. (1982). Social anxiety and assertiveness: a comparison of the correlations in phobic and college student samples. Behaviour Research and Therapy, 20(4), 403-404.

Chambless, D. (1985). The relationship with the severity of agoraphobia to associated psychopathology. Behaviour Research and Therapy, 23(3), 305-310.

Chien, H. C., Ku, C. H., Lu, R. B., Chu, H., Tao, Y. H. \& Chou, K. R. (2003). Effects of social skills training on improving social skills of patients with schizophrenia. Archives of Psychiatry Nursing, 17(5), 228-236.

Curran, J. P. (1977). Skills training as an approach to the treatment of hetero-social anxiety: A review. Psychological Bulletin, 84(1), 140-157.

Del Prette, A. \& Del Prette, Z. (2001). Psicologias das relações interpessoais: vivências para o trabalho em grupo. Petrópolis: Vozes.

Del Prette, Z. A. P. \& Del Prette, A. (2002). Transtornos psicológicos e habilidades sociais. Em H. J. Guilhardi, M. B. B. P. Madi, P. P. Queiroz \& M. C. Scoz (Orgs.), Sobre comportamento e cognição. Contribuições para a construção da teoria do comportamento. Santo André: ESETec.

Emmelkamp, P. M. \& Kuipers, A. C. (1979). Agoraphobia: a follow-up study four years after treatment. British Journal of Psychiatry, 134, 352-355.

Emmelkamp, P. M. (1980). Agoraphobic's interpersonal problems: their role in the effects of exposure in vivo therapy. Archives of General Psychiatry, 37(11), 1303-1306.

Emmelkamp, P. M. G., van den Hout, M. \& de Vries, K. (1983). Assertive training for agoraphobics. Behaviour Research and Therapy, 21(1), 63-68.

Fisher, L. M. \& Wilson, G. T. (1985). A study of the psychology of agoraphobia. Behaviour Research and Therapy, 23(2), 97-107.

Golstein, A. J. \& Chambless, D. (1978). A reanalysis of agoraphobia. Behavior Therapy, 9, 47-59.

Heimberg, R. G., Dodge, C. S., Hope, D. A., Kennedy, C. R., Zollo, L. J. \& Becker, R. E. (1990). Cognitive behavioral treatment for social phobia: comparison with a credible placebo control. Cognitive Therapy and Research, 14, 1-23.

Kashdan, T. B. \& Herbert, J. D. (2001). Social Anxiety Disorder in Childhood and adolescence: Current Status and Future Directions. Clinical Child and Family Psychology Review, 4(1), 37-61.
Lange, J. L. \& Jakubowski, P. (1976). Responsible assertive behavior. Illinois: Research Press.

Lucock, M. P. \& Salkovkis, P. M. (1988). Cognitive factors in social anxiety and its treatment. Behaviour Research and Therapy, 26(4), 297-302.

Marks, I. M. \& Gelder, M. G (1966). Different ages of onset in varieties of phobia. American Journal of Psychiatry, 123, 218-221.

Monti, P. M., Curran, J. P., Corriveau, D. P., DeLancey, A. L. \& Hagerman, S. M. (1980). Effects of social skills training groups and sensitivity training groups with psychiatric patients. Journal of Consulting and Clinical Psychology, 48(2), 241-248.

Patterson, T. L., Moscona, S., McKibbin, C. L., Davidson, K. \& Jeste, D. V. (2001). Social skills performance assessment among older patients with schizophrenia. Schizophrenia Research, 30, 48(2-3), 351-360

Pilkonis, P. A. (1977). The behavioral consequences of shyness. Journal of Personality, 45, 596-611.

Rapee, R. M. \& Lim, L. (1992). Discrepancy between self and observer ratings of performance in social phobics. Journal of Abnormal Psychology, 101(4), 728-731.

Rapee, R. M. \& Heimberg, R. G. (1997). A cognitive- behavioral model of anxiety in social phobia. Behaviour Research and Therapy, 35(8), 741-756.

Rapee, R. M. \& Spence, S. H. (2004). The etiology of social phobia: empirical evidence and an initial model. Clinical Psychology Review, 24(7), 737-767.

Segrin, C. (2000). Social skills deficits associated with depression. Clinical Psychology Review, 20(3), 379-403.

Segrin, C. \& Flora, J. (2000). Poor social skills are a vulnerability factor in the development of psychosocial problems. Human Communication Research, 26(3), 489-514.

Strahan, E. \& Conger, A. J. (1998). Social Anxiety and Its Effects on Performance and Perception. Journal of Anxiety Disorders, 12(4), 293-305.

Spence, S. H., Donovan, C. \& Toussaint, B. M. (1999). Social skills, social outcomes, and cognitive features of childhood social phobia. Journal of Abnormal Psychology, 108(2), 211-221.

Thompson, S. \& Rapee, R. M. (2002). The effect of situational structure on the social performance of socially anxious and non-anxious participants. Journal of Behavior Therapy and Experimental Psychiatry, 33(2), 91-102.

Turner, S. M., Beidel, D. C., Dancu, D. V. \& Keys, D. J. (1986). Psychopathology of social phobia and comparison to avoidant personality disorder. Journal of Abnormal Psychology, 95(4), 389-394.

Turner, S., Beidel, D., Cooley, M., Woody, S. R. \& Beidel, D. C. (1994). A multicomponent behavioral treatment for social phobia: social effectiveness therapy. Behaviour Research and Therapy, 32(4), 381-390.

Wenzel, A., Graff-Dolezal, J., Macho, M. \& Brendle, J. R. (2005). Communication and social skills in socially anxious and nonanxious individuals in the context of romantic relationships. Behaviour Research and Therapy, 43(4), 505-519.

Recebido em 26.07.2006

Primeira decisão editorial em 11.10.2006

Versão final em 27.07.2007

Aceito em 10.09.2007 